\title{
The Effect of Nitrogen Fertilizer Rates and Timing on Cranberry Yield and Fruit Quality
}

\author{
Joan R. Davenport \\ Ocean Spray Cranberries, Inc., One Ocean Spray Drive, Lakeville-Middleboro, MA 02349 \\ Additional index words. organic soils, TAcy, fruit rot
}

\begin{abstract}
To examine the impact of $\mathbf{N}$ fertilizer rates and timing on cranberry (Vaccinium macrocarpon Ait.) yield and the fruit quality factors total anthocyanin, average berry weight, and field and storage rot, plots were established for 3 to 4 years. The cranberries received a total of 0,22 , or $44 \mathrm{~kg}$ N/ha over the growing season applied in three, four, or five applications, which varied by growth stage. There were eleven possible treatment combinations in Massachusetts on 'Early Black' and 'Howes' and in Wisconsin on 'Stevens' and 'Searles', and seven possible treatments in New Jersey on 'Early Black' and in Washington on 'McFarlin'. The results showed a nationwide response to $\mathbf{N}$ that suggests the experimental middle rate of $22 \mathrm{~kg} \cdot \mathrm{ha}^{-1}$ would result in high yields with moderate rot. However, the best timing for applying the fertilizer varied by both state and cultivar, where three late season applications were best on 'Early Black' in New Jersey versus four early season applications on the same cultivar in Massachusetts, and applying $\mathbf{N}$ fertilizers across five applications was optimal for 'Stevens' in Wisconsin.
\end{abstract}

The American cranberry (Vaccinium macrocarpon) is a lowgrowing, nondeciduous woody perennial that sets fruit buds before overwintering. It is grown in a diversity of conditions throughout North America on commercial cranberry farms. The soil conditions on these sites ranges from high organic peat and muck soils to sandy mineral soils (Davenport and DeMoranville, 1993).

Over the past 25 years, there has been a yield increase in commercial cranberry beds. Average production in 1965 was 7.8 $\mathrm{Mg} \cdot \mathrm{ha}^{-1}$ (Peterson et al., 1968). By 1990, the North American average was $16.8 \mathrm{Mg} \cdot \mathrm{ha}^{-1}$ (U.S. Dept. of Agriculture, 1990). Concomitant with the rise in yield has been a rise in the use of farm chemicals, fertilizers being among these (Deubert and Caruso, 1989).

Early studies of the impact of fertilizers on cranberry yield focused on blended fertilizers. When these studies were conducted, little to no fertilizer was regularly used by commercial farmers (Beckwith, 1919). Although these studies helped provide guidelines for cranberry farmers for fertilizer use, there still was little understanding of the impact of a given nutrient on the crop.

With the increased value of the cranberry crop, cranberry farmers began increasing their fertilizer applications to increase productivity. Today, the variability in fertilizer use between cranberry farmers is almost as great as the number of farmers. A study conducted on cranberries in Oregon has shown that different optimum rates and timings of $\mathrm{N}$ for 'Crowley' and 'Stevens' (Hart et al., 1990).

Nitrogen has been implicated in other studies on cranberries as an element associated with increases in crop yield (Eaton, 1971). Additionally, evidence indicates that excess $\mathrm{N}$ can lead to excessive vegetative production and, concurrently, reduce crop yield and quality (Eck, 1976). The relationship with fruit quality, particularly fruit rot, is of great importance for fresh fruit storagability and marketing. This study was conducted to establish an optimal rate and timing strategy for $\mathrm{N}$ fertilizer use in cranberries.

Received for publication 1 Sept. 1995. Accepted for publication 27 June 1996. The cost of publishing this paper was defrayed in part by the payment of page charges. Under postal regulations, this paper therefore must be hereby marked advertisement solely to indicate this fact.

\section{Materials and Methods}

In 1989, field trials were set up on two commercial cranberry farms in Massachusetts. On each farm, sets of plots were established on 'Howes' and 'Early Black', late and early producing cultivars, respectively. Blocks of plots containing $1002 \times 2-\mathrm{m}$ plots were measured and marked with pin flags. Treatment assignments were completely random. The experiment consisted of 11 treatments to provide either 0,22 , or $44 \mathrm{~kg} \mathrm{~N} / \mathrm{ha}$ over three, four, or five applications (Table 1), which were replicated six times. The number of plots in the block exceeded the total number needed so that unsuitable plots (e.g., plots with excessive weeds, lack of vine cover) could be avoided.

Nitrogen was applied as ammonium sulfate in the form of the commercial fertilizer 21-0-0. This was applied to each plot at the designated time with the use of a 500-mL plastic container with holes drilled into the lid. This shaker cup was used to sprinkle the fertilizer evenly throughout the plot. Application times were by growth stage and the following growth stages were used: budbreak, peak bloom, fruit set, bud set, and preharvest.

Each entire block of plots was also supplied with $66 \mathrm{~kg} \cdot \mathrm{ha}^{-1} \mathrm{~K}$ and $\mathrm{P}$ fertilizers as commercial triple superphosphate (0-46-0) and potassium sulfate $(0-0-50)$. The $\mathrm{P}$ and $\mathrm{K}$ applications were split across the five possible timings and were broadcast with a Cyclone spreader.

Fertilizer experiments were also initiated in 1990 in New Jersey, Wisconsin, and Washington. Table 2 lists the sites and cultivars per each growing area. The treatments were modified slightly for New Jersey and Washington, removing the preharvest fertilizer application (Table 1) to accommodate lower average yields and higher organic matter soils in those regions (Davenport and DeMoranville, 1993). Additionally, the number of replicates of each treatment was reduced to five. The total block of plots was reduced also, to $642 \times 2-\mathrm{m}$ plots in Wisconsin and 42 in New Jersey and Washington. Phosphorus and potassium application amounts were held uniform, but applied over one less application in Washington and New Jersey.

At the end of each growing season, fruit was hand harvested from two $900-\mathrm{cm}^{2}$ areas in each plot. The fruit from each $900-\mathrm{cm}^{2}$ area (the sample) were weighed to determine yield. A subsample of 100 random fruit was taken from each sample. The subsample was weighed to determine the average berry weight and evaluated for 
Table 1. Rates and timings (by growth stage) of $\mathrm{N}$ fertilizer applications and the states where the treatments were used.

\begin{tabular}{lclc}
\hline \hline $\begin{array}{l}\mathrm{N} \\
\left(\mathrm{kg} \cdot \mathrm{ha}^{-1}\right)\end{array}$ & $\begin{array}{c}\text { Aplications } \\
(\text { no. })\end{array}$ & \multicolumn{1}{c}{$\begin{array}{c}\text { Times } \\
\text { applied }\end{array}$} \\
\hline 0 & Not applicable & Not applicable & State \\
22 & 5 & Budbreak, peak bloom, fruit set, bud set, preharvest & MA, NJ, WA, WI \\
44 & 5 & Budbreak, peak bloom, fruit set, bud set, preharvest & MA, WI \\
22 & 4 & Peak bloom, fruit set, bud set, preharvest & MA, WI \\
44 & 4 & Peak bloom, fruit set, bud set, preharvest & MA, WI \\
22 & 4 & Budbreak, peak bloom, fruit set, bud set & MA, WI \\
44 & 4 & Budbreak, peak bloom, fruit set, bud set & MA, WA, WI \\
22 & 3 & Peak bloom, fruit set, bud set & MA, NJ, WA, WI \\
44 & 3 & Peak bloom, fruit set, bud set & MA, NJ, WA, WI \\
22 & 3 & Budbreak, peak bloom, fruit set & MA, NJ, WA, WI \\
44 & 3 & Budbreak, peak bloom, fruit set & MA, NJ, WA, WI \\
\hline
\end{tabular}

soundness to determine the percentage fruit rot. All samples were then stored at $4{ }^{\circ} \mathrm{C}$. After 30 - and 60-d intervals, the fruit were reevaluated for rot. At the end of $60 \mathrm{~d}$, the two original samples from each plot were combined and a subsample of nonrotted fruit was taken. This subsample was frozen and analyzed for total anthocyanin (TAcy) as a measure of fruit color (Fuleki and Francis, 1968).

The experiment was continued for 3 years in all locations but one. One of the Massachusetts sites (Bogs 2 and 4) had been saltwater flooded during Hurricane Bob on 19 Aug. 1991. The damage to the fruit at harvest was so severe that the fruit could not be accurately evaluated. The experiment was continued for a further growing season. Only Bog 2 with 'Early Black' was harvested from this site in 1992. Several replicates were not harvested because salt damage from the previous year had destroyed the growth. The cranberry farmer had harvested 'Howes' without notice before our harvest of Bog 4.
Data were analyzed using PROC GLM on PC SAS (SAS Institute, Cary, N.C.). Data from each year was analyzed separately to account for differences in climatic factors in each year.

\section{Results and Discussion}

Average berry weight and TAcy. There was no overall impact of $\mathrm{N}$ rates or timing on average berry weight or TAcy. In a few isolated instances there was a significant differences in each of these parameters based on either level of $\mathrm{N}$ applied or a combination of rate and timing. These instances were not consistent from year to year and do not provide any insights.

Nitrogen rates. The data from the years 1991 and 1992 were tabulated by rates of $\mathrm{N}$ applied and analyzed using a backwards stepwise regression procedure. After determining that yield and fruit rot varied by year and location, the data were sorted by year and location of plots-a combination cultivar and commercial

Table 2. Location of sites, cultivars studied, 5-year yield averages (1988-92), and the years in which the N fertilizer treatments were applied.

\begin{tabular}{llccc}
\hline \hline $\begin{array}{l}\text { Bog } \\
\text { (no.) }\end{array}$ & State & Cultivar & Years & $\begin{array}{c}\text { 5-Year yield avg } \\
\left(\mathrm{Mg}^{-1}\right)^{-1}\end{array}$ \\
\hline 1 & MA & Early Black & $1989-91$ & 11.71 \\
2 & MA & Early Black & $1989-92^{z}$ & 16.54 \\
3 & MA & Howes & $1989-91$ & 19.32 \\
4 & MA & Howes & $1989-92^{z}$ & 20.56 \\
5 & NJ & Early Black & $1990-92$ & 9.47 \\
6 & NJ & Early Black & $1990-92$ & 20.62 \\
7 & WA & McFarlin & $1990-92$ & 11.95 \\
8 & WI & Stevens & $1990-92$ & 19.54 \\
9 & WI & Searles & 16.26 \\
10 & WI & Searles $^{\mathrm{y}}$ & $1990-92$ & 17.34 \\
\hline
\end{tabular}

${ }^{\mathrm{z} P l o t s}$ not harvested at one location in 1992 due to salt damage from flood. Experiment continued at this site a fourth year; however, 'Howes' were commercially harvested before scheduled experimental harvest, therefore, data were lost.

'Bogs 9 and 10 differed by soil type: Bog 9 was a mineral-based soil and Bog 10 was an organic soil.

Table 3. Cranberry yield and fruit rot levels after at least 3 years of receiving 0,22 , or $44 \mathrm{~kg} \mathrm{~N} / \mathrm{ha}$.

\begin{tabular}{|c|c|c|c|c|}
\hline \multirow{2}{*}{$\begin{array}{l}\mathrm{N} \\
\left(\mathrm{kg} \cdot \mathrm{ha}^{-1}\right)\end{array}$} & \multirow{2}{*}{$\begin{array}{c}\text { Yield } \\
\left(\mathrm{Mg} \cdot \mathrm{ha}^{-1}\right)\end{array}$} & \multirow{2}{*}{$\begin{array}{l}\text { Field rot } \\
\qquad(\%)\end{array}$} & \multicolumn{2}{|c|}{ Storage rot $(\%)$} \\
\hline & & & $30 \mathrm{~d}$ & $60 \mathrm{~d}$ \\
\hline 0 & 12.78 & 3.82 & 5.38 & 8.16 \\
\hline 22 & 16.55 & 5.45 & 6.56 & 9.70 \\
\hline 44 & 14.55 & 7.74 & 10.17 & 14.16 \\
\hline Significance & $\mathrm{L}^{*} \mathrm{Q}^{*}$ & $\mathrm{~L}^{* * * *} \mathrm{Q}^{* * * *}$ & $\mathrm{~L}^{* * * *} \mathrm{Q}^{* * * *}$ & $\mathrm{~L}^{* * * *} \mathrm{Q}^{* * * *}$ \\
\hline
\end{tabular}

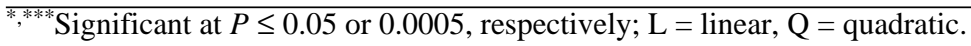


grower - to evaluate the trends. The data were evaluated using linear and quadratic contrasts with SAS PROC GLM. For this section, rates of $\mathrm{N}$ will be referred to as $\mathrm{kg} \mathrm{N}$ applied.

In 1992, at the end of the third harvest, yield and fruit rot showed an impact from $\mathrm{N}$ (Table 3). Yield was highest with $22 \mathrm{~kg} \mathrm{~N}$. Field rot and 30 and 60 storage rots were increased by $\mathrm{N}$ (Table 3 ). Field rot increased with increasing $\mathrm{N}$ rates. Fruit rot increased after 30 and $60 \mathrm{~d}$ of cold storage, but in both cases, rot increased when $\mathrm{N}$ rates increased. Yield was influenced by the rate of $\mathrm{N}$ in 10 of the 15 possible year and location combinations (Table 4). In the 10 instances when yield was related to $\mathrm{N}$ rate, the response included a quadratic term, reflecting that yield did not simply increase with increasing $\mathrm{N}$ fertilizer additions.

Fruit rot (field and storage rots) was related to level of $\mathrm{N}$ (Table 4). The trend at $60 \mathrm{~d}$ echoes that at $30 \mathrm{~d}$, so the data are not shown. In all cases, the lowest rot was at the $0 \mathrm{~N}$ level and highest at 44 . The relationship between fruit rot and $\mathrm{N}$ rate showed a linear trend only in five cases, indicating an overall rise in rot as $\mathrm{N}$ rate increased. In 13 of the bog and year combinations, there was also a quadratic response of rot to $\mathrm{N}$ rate. The average rot levels when a quadratic response occurred are for a larger increase in rot between the 22 and $44 \mathrm{~N}$ rates that between the 0 and 22 rates.

Overall, based on $\mathrm{N}$ rates alone, the data suggest that yield is optimized with $22 \mathrm{~kg} \mathrm{~N} / \mathrm{ha}$. This rate also has only a moderate amount of rot, at harvest and after $30 \mathrm{~d}$ storage. This indicates that $22 \mathrm{~kg} \mathrm{~N} / \mathrm{ha}$ could be used as an optimal rate across regions and cultivars.

Nitrogen rates and timings. The timing of the fertilizer applications alone was not related to any of the parameters evaluated. However, a treatment effect (rate and timing) was found on all sites for some parameters.

For several bogs, yields were highest when no $\mathrm{N}$ was applied. This was found in 'Howes' in Massachusetts and 'McFarlin' in Washington (Table 5). At one location of 'Early Black' in New Jersey (Bog 5), for 2 consecutive years the $0 \mathrm{~N}$ treatment also showed a high yield. In all three of these cases, the beds are poor

Table 4. Cranberry yield, field rot, and storage rot after $30 \mathrm{~d}$ cold storage for cranberries receiving three different rates of total $\mathrm{N}$ over the growing season. Lack of data for Bogs 1 and 3 in 1992 are because the study for that site ended in 1991; for Bog 2 in 1991 missing data reflect fruit damage due to a hurricane.

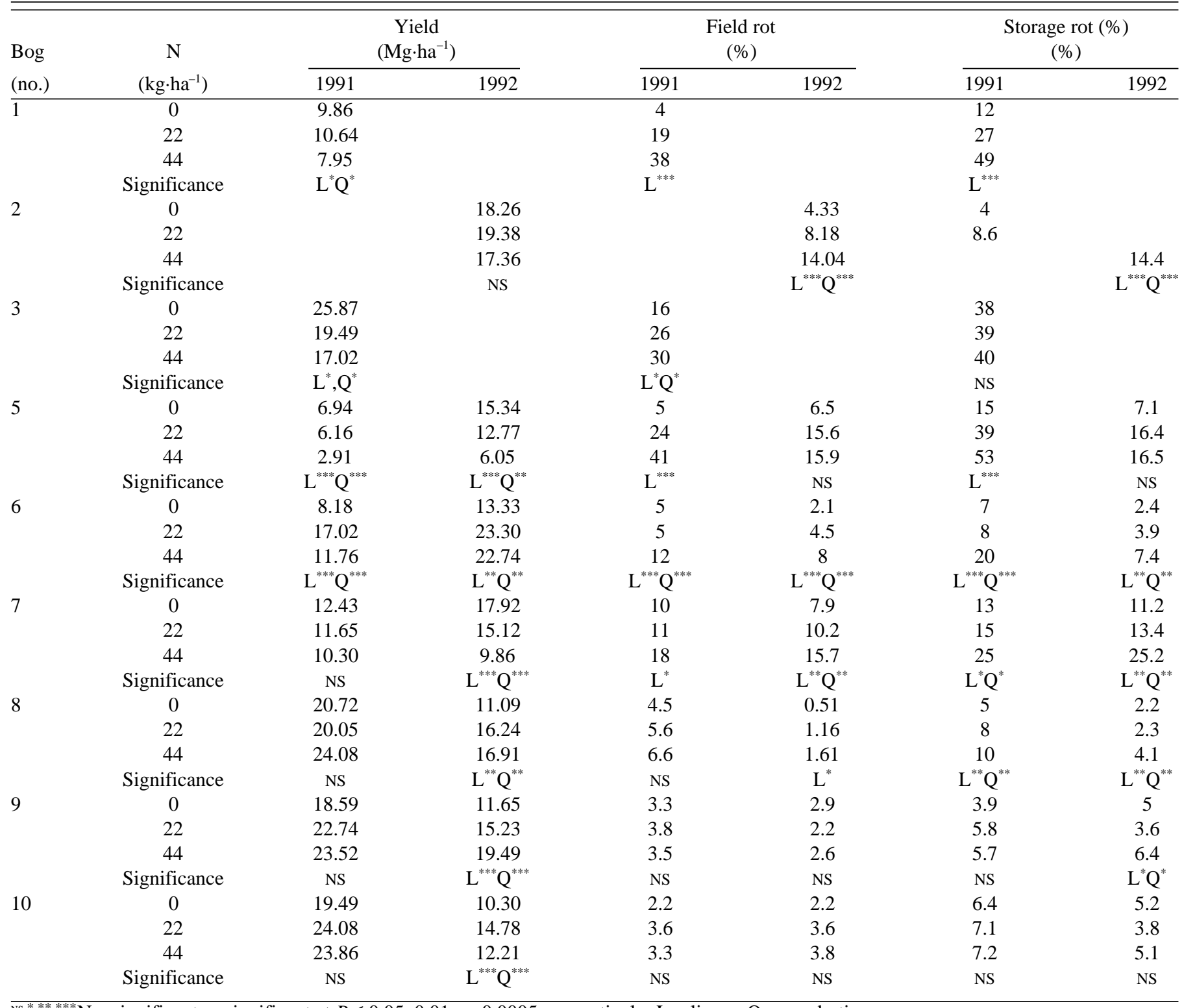

$\mathrm{NS}^{*},{ }^{* *},{ }^{* * *}$ Nonsignificant or significant at $P \leq 0.05,0.01$, or 0.0005 , respectively; $\mathrm{L}=$ linear, $\mathrm{Q}=$ quadratic. 
Table 5. Average \pm standard deviations for cranberry yield, field rot, and storage rot after $30 \mathrm{~d}$ cold storage for cranberries receiving three different rates of total $\mathrm{N}$ at different timings in the growing season. Lack of data for Bog 2 in 1991 reflect fruit damage due to a hurricane.

\begin{tabular}{|c|c|c|c|c|c|c|c|c|}
\hline \multirow{2}{*}{$\begin{array}{l}\text { Bog } \\
\text { (no.) }\end{array}$} & \multirow{2}{*}{$\begin{array}{c}\mathrm{N} \\
\left(\mathrm{kg} \cdot \mathrm{ha}^{-1}\right)\end{array}$} & \multirow{2}{*}{$\begin{array}{c}\text { Time } \\
\text { applied }^{\mathrm{z}}\end{array}$} & \multicolumn{2}{|c|}{$\begin{array}{c}\text { Yield } \\
\left(\mathrm{Mg} \cdot \mathrm{ha}^{-1}\right)\end{array}$} & \multicolumn{2}{|c|}{$\begin{array}{c}\text { Field rot } \\
(\%)\end{array}$} & \multicolumn{2}{|c|}{$\begin{array}{c}\text { Storage rot } \\
(\%)\end{array}$} \\
\hline & & & 2nd yr & $3 \mathrm{rd} \mathrm{yr}$ & $2 \mathrm{nd} \mathrm{yr}$ & $3 \mathrm{rd} \mathrm{yr}$ & $2 \mathrm{nd} \mathrm{yr}$ & $3 \mathrm{rd} \mathrm{yr}$ \\
\hline \multirow[t]{12}{*}{$\overline{1}$} & 0 & ALL & $8.18 \pm 2.14$ & $9.86 \pm 1.32$ & $4 \pm 1$ & $3 \pm 2$ & $3 \pm 2$ & $12 \pm 8$ \\
\hline & 22 & ALL & $11.42 \pm 2.06$ & $10.42 \pm 7.18$ & $19 \pm 4$ & $20 \pm 5$ & $15 \pm 8$ & $27 \pm 11$ \\
\hline & 44 & ALL & $7.73 \pm 2.32$ & $7.50 \pm 4.45$ & $23 \pm 12$ & $32 \pm 17$ & $24 \pm 13$ & $47 \pm 19$ \\
\hline & 22 & $4 \mathrm{~L}$ & $13.78 \pm 8.12$ & $10.64 \pm 3.01$ & $8 \pm 5$ & $18 \pm 7$ & $11 \pm 7$ & $27 \pm 8$ \\
\hline & 44 & $4 \mathrm{~L}$ & $13.22 \pm 2.42$ & $9.18 \pm 5.06$ & $18 \pm 7$ & $33 \pm 12$ & $20 \pm 5$ & $43 \pm 7$ \\
\hline & 22 & $4 \mathrm{E}$ & $15.90 \pm 4.98$ & $8.29 \pm 4.09$ & $5 \pm 4$ & $15 \pm 9$ & $6 \pm 4$ & $26 \pm 9$ \\
\hline & 44 & $4 \mathrm{E}$ & $11.09 \pm 1.65$ & $7.17 \pm 6.77$ & $25 \pm 5$ & $44 \pm 15$ & $26 \pm 8$ & $54 \pm 17$ \\
\hline & 22 & $3 \mathrm{~L}$ & $12.21 \pm 2.33$ & $14.45 \pm 2.18$ & $10 \pm 3$ & $22 \pm 7$ & $9 \pm 4$ & $28 \pm 13$ \\
\hline & 44 & $3 \mathrm{~L}$ & $7.50 \pm 2.05$ & $8.62 \pm 2.94$ & $19 \pm 5$ & $35 \pm 12$ & $21 \pm 8$ & $40 \pm 12$ \\
\hline & 22 & $3 \mathrm{E}$ & $14.11 \pm 4.09$ & $8.96 \pm 4.00$ & $11 \pm 4$ & $19 \pm 12$ & $12 \pm 9$ & $22 \pm 10$ \\
\hline & 44 & $3 \mathrm{E}$ & $9.97 \pm 2.03$ & $7.50 \pm 3.80$ & $21 \pm 2$ & $48 \pm 15$ & $24 \pm 4$ & $63 \pm 13$ \\
\hline & Significance & & $*$ & NS & $* * *$ & $* * *$ & $* * *$ & $* * *$ \\
\hline \multirow[t]{12}{*}{2} & 0 & ALL & & $18.26 \pm 2.98$ & & $4 \pm 2$ & & $4 \pm 1$ \\
\hline & 22 & ALL & & $16.91 \pm 4.67$ & & $5 \pm 3$ & & $6 \pm 3$ \\
\hline & 44 & ALL & & $16.02 \pm 5.88$ & & $13 \pm 4$ & & $14 \pm 4$ \\
\hline & 22 & $4 \mathrm{~L}$ & & $19.26 \pm 5.93$ & & $7 \pm 3$ & & $9 \pm 5$ \\
\hline & 44 & $4 \mathrm{~L}$ & & $23.52 \pm 2.57$ & & $12 \pm 4$ & & $12 \pm 5$ \\
\hline & 22 & $4 \mathrm{E}$ & & $17.58 \pm 3.75$ & & $10 \pm 5$ & & $10 \pm 3$ \\
\hline & 44 & $4 \mathrm{E}$ & & $17.25 \pm 5.00$ & & $14 \pm 6$ & & $15 \pm 2$ \\
\hline & 22 & $3 \mathrm{~L}$ & & $19.49 \pm 4.10$ & & $11 \pm 7$ & & $11 \pm 8$ \\
\hline & 44 & $3 \mathrm{~L}$ & & $16.13 \pm 4.49$ & & $15 \pm 7$ & & $15 \pm 4$ \\
\hline & 22 & $3 \mathrm{E}$ & & $23.52 \pm 4.05$ & & $7 \pm 3$ & & $7 \pm 2$ \\
\hline & 44 & $3 \mathrm{E}$ & & $14.45 \pm 4.26$ & & $16 \pm 4$ & & $17 \pm 6$ \\
\hline & Significance & & & NS & & $*$ & & $* * *$ \\
\hline \multirow[t]{12}{*}{3} & 0 & ALL & $13.89 \pm 8.97$ & $25.98 \pm 14.43$ & $8 \pm 8$ & $16 \pm 6$ & $9 \pm 13$ & $38 \pm 22$ \\
\hline & 22 & ALL & $9.63 \pm 4.65$ & $20.72 \pm 11.21$ & $11 \pm 7$ & $21 \pm 10$ & $11 \pm 8$ & $30 \pm 13$ \\
\hline & 44 & ALL & $4.82 \pm 1.88$ & $17.92 \pm 5.87$ & $14 \pm 7$ & $32 \pm 6$ & $16 \pm 7$ & $47 \pm 6$ \\
\hline & 22 & $\mathrm{AL}$ & $8.96 \pm 5.90$ & $16.24 \pm 10.35$ & $12 \pm 8$ & $26 \pm 12$ & $13 \pm 12$ & $43 \pm 13$ \\
\hline & 44 & $4 \mathrm{~L}$ & $6.72 \pm 3.68$ & $20.38 \pm 8.36$ & $15 \pm 8$ & $24 \pm 12$ & $19 \pm 11$ & $32 \pm 9$ \\
\hline & 22 & $4 \mathrm{E}$ & $10.08 \pm 5.76$ & $15.79 \pm 7.02$ & $10 \pm 6$ & $32 \pm 14$ & $13 \pm 8$ & $51 \pm 17$ \\
\hline & 44 & $4 \mathrm{E}$ & $7.84 \pm 2.93$ & $17.81 \pm 3.92$ & $13 \pm 12$ & $29 \pm 14$ & $21 \pm 13$ & $49 \pm 13$ \\
\hline & 22 & $3 \mathrm{~L}$ & $8.18 \pm 3.75$ & $24.08 \pm 10.16$ & $9 \pm 7$ & $26 \pm 11$ & $13 \pm 10$ & $34 \pm 11$ \\
\hline & 44 & $3 \mathrm{~L}$ & $6.72 \pm 3.65$ & $13.89 \pm 5.71$ & $13 \pm 11$ & $29 \pm 11$ & $15 \pm 8$ & $31 \pm 8$ \\
\hline & 22 & $3 \mathrm{E}$ & $5.71 \pm 1.08$ & $20.83 \pm 9.80$ & $10 \pm 7$ & $28 \pm 14$ & $12 \pm 8$ & $38 \pm 16$ \\
\hline & 44 & $3 \mathrm{E}$ & $5.49 \pm 2.63$ & $15.01 \pm 6.09$ & $15 \pm 6$ & $33 \pm 12$ & $17 \pm 12$ & $45 \pm 15$ \\
\hline & Significance & $* *$ & NS & NS & NS & NS & NS & \\
\hline \multirow{8}{*}{5} & 0 & ALL & $6.94 \pm 4.91$ & $16.46 \pm 6.96$ & $5 \pm 3$ & $7 \pm 6$ & $15 \pm 4$ & $7 \pm 5$ \\
\hline & 22 & ALL & $3.58 \pm 2.34$ & $12.32 \pm 4.06$ & $25 \pm 12$ & $14 \pm 14$ & $39 \pm 14$ & $14 \pm 15$ \\
\hline & 44 & ALL & $5.26 \pm 3.29$ & $8.62 \pm 6.96$ & $46 \pm 24$ & $18 \pm 7$ & $59 \pm 11$ & $19 \pm 9$ \\
\hline & 22 & $3 \mathrm{~L}$ & $5.94 \pm 1.99$ & $14.00 \pm 8.65$ & $30 \pm 12$ & $18 \pm 8$ & $41 \pm 10$ & $20 \pm 9$ \\
\hline & 44 & $3 \mathrm{~L}$ & $2.69 \pm 1.22$ & $2.46 \pm 1.40$ & $36 \pm 11$ & $13 \pm 13$ & $54 \pm 13$ & $13 \pm 6$ \\
\hline & 22 & $3 \mathrm{E}$ & $7.50 \pm 2.90$ & $11.89 \pm 4.01$ & $16 \pm 9$ & $15 \pm 8$ & $35 \pm 16$ & $15 \pm 12$ \\
\hline & 44 & $3 \mathrm{E}$ & $2.35 \pm 0.52$ & $7.06 \pm 5.48$ & $42 \pm 19$ & $16 \pm 16$ & $45 \pm 20$ & $18 \pm 16$ \\
\hline & Significance & $*$ & $* *$ & $* * *$ & NS & $* * *$ & NS & \\
\hline \multirow[t]{8}{*}{6} & 0 & ALL & $8.29 \pm 2.37$ & $13.33 \pm 3.17$ & $5 \pm 2$ & $2 \pm 1$ & $7 \pm 2$ & $2 \pm 1$ \\
\hline & 22 & ALL & $15.90 \pm 5.58$ & $23.74 \pm 3.94$ & $5 \pm 3$ & $6 \pm 6$ & $8 \pm 6$ & $5 \pm 6$ \\
\hline & 44 & ALL & $14.00 \pm 7.04$ & $26.66 \pm 6.16$ & $10 \pm 5$ & $10 \pm 4$ & $15 \pm 6$ & $10 \pm 6$ \\
\hline & 22 & $3 \mathrm{~L}$ & $17.47 \pm 4.69$ & $22.18 \pm 8.01$ & $8 \pm 3$ & $5 \pm 4$ & $12 \pm 4$ & $4 \pm 3$ \\
\hline & 44 & $3 \mathrm{~L}$ & $9.30 \pm 3.93$ & $20.83 \pm 2.85$ & $13 \pm 6$ & $7 \pm 8$ & $21 \pm 9$ & $6 \pm 7$ \\
\hline & 22 & $3 \mathrm{E}$ & $17.81 \pm 2.88$ & $24.08 \pm 5.75$ & $3 \pm 2$ & $2 \pm 1$ & $4 \pm 3$ & $3 \pm 1$ \\
\hline & 44 & $3 \mathrm{E}$ & $11.87 \pm 4.60$ & $20.61 \pm 5.78$ & $11 \pm 6$ & $7 \pm 5$ & $23 \pm 10$ & $6 \pm 4$ \\
\hline & Significance & $* *$ & $* *$ & $* * *$ & NS & $* * *$ & NS & \\
\hline \multirow[t]{4}{*}{7} & 0 & ALL & $12.43 \pm 2.49$ & $17.92 \pm 2.29$ & $10 \pm 7$ & $8 \pm 8$ & $14 \pm 8$ & $11 \pm 9$ \\
\hline & 22 & ALL & $12.10 \pm 2.83$ & $14.70 \pm 4.26$ & $9 \pm 4$ & $7 \pm 5$ & $13 \pm 7$ & $8 \pm 6$ \\
\hline & 44 & ALL & $10.30 \pm 3.98$ & $9.74 \pm 4.76$ & $16 \pm 15$ & $17 \pm 9$ & $27 \pm 17$ & $27 \pm 12$ \\
\hline & 22 & $3 \mathrm{~L}$ & $10.86 \pm 3.81$ & $16.46 \pm 3.74$ & $11 \pm 6$ & $13 \pm 8$ & $16 \pm 10$ & $19 \pm 11$ \\
\hline
\end{tabular}


Table 5. Continued.

\begin{tabular}{|c|c|c|c|c|c|c|c|c|}
\hline \multirow{2}{*}{$\begin{array}{l}\text { Bog } \\
\text { (no.) }\end{array}$} & \multirow{2}{*}{$\begin{array}{c}\mathrm{N} \\
\left(\mathrm{kg} \cdot \mathrm{ha}^{-1}\right)\end{array}$} & \multirow{2}{*}{$\begin{array}{c}\text { Time } \\
\text { applied }^{\mathrm{z}}\end{array}$} & \multicolumn{2}{|c|}{ 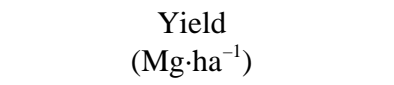 } & \multicolumn{2}{|c|}{$\begin{array}{l}\text { Field rot } \\
\qquad(\%)\end{array}$} & \multicolumn{2}{|c|}{$\begin{array}{c}\text { Storage rot } \\
(\%)\end{array}$} \\
\hline & & & 2nd yr & $3 \mathrm{rd} \mathrm{yr}$ & $2 \mathrm{nd} y r$ & $3 \mathrm{rd} \mathrm{yr}$ & $2 \mathrm{nd} \mathrm{yr}$ & $3 \mathrm{rd} \mathrm{yr}$ \\
\hline \multirow[t]{4}{*}{7} & 44 & $3 \mathrm{~L}$ & $10.30 \pm 3.57$ & $10.42 \pm 4.89$ & $18 \pm 8$ & $16 \pm 5$ & $23 \pm 8$ & $28 \pm 15$ \\
\hline & 22 & $3 \mathrm{E}$ & $11.87 \pm 4.78$ & $14.11 \pm 2.45$ & $14 \pm 7$ & $10 \pm 6$ & $17 \pm 8$ & $13 \pm 7$ \\
\hline & 44 & $3 \mathrm{E}$ & $10.19 \pm 5.62$ & $9.63 \pm 6.47$ & $19 \pm 16$ & $15 \pm 10$ & $25 \pm 16$ & $20 \pm 10$ \\
\hline & Significance & NS & $*$ & NS & NS & NS & $*$ & \\
\hline \multirow[t]{12}{*}{8} & 0 & ALL & $20.72 \pm 2.38$ & $10.30 \pm 3.54$ & $4 \pm 4$ & $0.5 \pm 0.3$ & $5 \pm 4$ & $2.3 \pm 1.3$ \\
\hline & 22 & ALL & $21.17 \pm 4.34$ & $16.80 \pm 1.88$ & $5 \pm 1$ & $0.8 \pm 0.6$ & $6 \pm 1$ & $1.4 \pm 0.9$ \\
\hline & 44 & ALL & $22.74 \pm 8.23$ & $13.66 \pm 3.54$ & $10 \pm 3$ & $1.3 \pm 0.9$ & $13 \pm 3$ & $1.9 \pm 0.8$ \\
\hline & 22 & $4 \mathrm{~L}$ & $19.49 \pm 7.32$ & $15.01 \pm 3.54$ & $8 \pm 3$ & $0.7 \pm 0.5$ & $11 \pm 4$ & $1.3 \pm 0.8$ \\
\hline & 44 & $4 \mathrm{~L}$ & $22.74 \pm 5.87$ & $14.22 \pm 1.99$ & $5 \pm 2$ & $0.8 \pm 0.3$ & $8 \pm 3$ & $3.4 \pm 2.1$ \\
\hline & 22 & $4 \mathrm{E}$ & $21.26 \pm 8.10$ & $13.66 \pm 2.71$ & $4 \pm 4$ & $0.9 \pm 0.5$ & $9 \pm 6$ & $2.4 \pm 1.3$ \\
\hline & 44 & $4 \mathrm{E}$ & $27.66 \pm 6.82$ & $10.86 \pm 3.08$ & $6 \pm 3$ & $0.8 \pm 0.5$ & $10 \pm 3$ & $3.8 \pm 2.9$ \\
\hline & 22 & $3 \mathrm{~L}$ & $16.91 \pm 4.73$ & $15.34 \pm 3.30$ & $6 \pm 2$ & $0.9 \pm 0.1$ & $9 \pm 4$ & $2.4 \pm 1.6$ \\
\hline & 44 & $3 \mathrm{~L}$ & $21.62 \pm 4.33$ & $13.78 \pm 2.51$ & $4 \pm 2$ & $1.1 \pm 0.9$ & $8 \pm 2$ & $8.1 \pm 4.4$ \\
\hline & 22 & $3 \mathrm{E}$ & $21.73 \pm 6.63$ & $12.66 \pm 5.04$ & $4 \pm 3$ & $1.0 \pm 0.9$ & $7 \pm 5$ & $3.4 \pm 2.0$ \\
\hline & 44 & $3 \mathrm{E}$ & $25.87 \pm 3.62$ & $8.51 \pm 1.61$ & $8 \pm 5$ & $0.9 \pm 0.4$ & $10 \pm 3$ & $3.0 \pm 1.6$ \\
\hline & Significance & NS & $* * *$ & $*$ & NS & $*$ & $* * *$ & \\
\hline \multirow[t]{12}{*}{9} & 0 & ALL & $18.59 \pm 4.15$ & $11.09 \pm 2.64$ & $3 \pm 1$ & $3 \pm 2$ & $4 \pm 1$ & $5 \pm 2$ \\
\hline & 22 & ALL & $21.28 \pm 5.78$ & $15.79 \pm 5.88$ & $3 \pm 1$ & $4 \pm 2$ & $6 \pm 1$ & $5 \pm 2$ \\
\hline & 44 & ALL & $21.62 \pm 5.48$ & $15.57 \pm 4.57$ & $3 \pm 1$ & $2 \pm 0.5$ & $5 \pm 1$ & $5 \pm 2$ \\
\hline & 22 & $4 \mathrm{~L}$ & $20.61 \pm 11.88$ & $15.01 \pm 1.60$ & $5 \pm 3$ & $2 \pm 1$ & $6 \pm 3$ & $3 \pm 1$ \\
\hline & 44 & $4 \mathrm{~L}$ & $27.22 \pm 6.14$ & $16.58 \pm 5.17$ & $3 \pm 1$ & $3 \pm 1$ & $6 \pm 1$ & $7 \pm 3$ \\
\hline & 22 & $4 \mathrm{E}$ & $20.16 \pm 6.36$ & $18.82 \pm 3.40$ & $3 \pm 1$ & $2 \pm 1$ & $5 \pm 1$ & $3 \pm 0.5$ \\
\hline & 44 & $4 \mathrm{E}$ & $24.53 \pm 1.5$ & $17.02 \pm 1.97$ & $5 \pm 0.5$ & $3 \pm 2$ & $7 \pm 1$ & $5 \pm 2$ \\
\hline & 22 & $3 \mathrm{~L}$ & $24.42 \pm 5.34$ & $16.46 \pm 2.79$ & $4 \pm 1$ & $2 \pm 1$ & $6 \pm 2$ & $3 \pm 2$ \\
\hline & 44 & $3 \mathrm{~L}$ & $20.16 \pm 2.93$ & $19.49 \pm 4.80$ & $2 \pm 1$ & $3 \pm 3$ & $4 \pm 1$ & $9 \pm 7$ \\
\hline & 22 & $3 \mathrm{E}$ & $27.33 \pm 3.21$ & $14.90 \pm 4.20$ & $4 \pm 1$ & $1 \pm 0.5$ & $6 \pm 1$ & $3 \pm 1$ \\
\hline & 44 & $3 \mathrm{E}$ & $24.19 \pm 6.14$ & $16.24 \pm 2.25$ & $4 \pm 2$ & $2 \pm 1$ & $7 \pm 3$ & $6 \pm 3$ \\
\hline & Significance & NS & NS & NS & NS & $* *$ & $* *$ & \\
\hline \multirow[t]{12}{*}{10} & 0 & ALL & $19.49 \pm 5.47$ & $11.65 \pm 3.30$ & $2 \pm 1$ & $4 \pm 2$ & $6 \pm 1$ & $5 \pm 2$ \\
\hline & 22 & ALL & $23.97 \pm 5.90$ & $18.70 \pm 7.61$ & $3 \pm 3$ & $2 \pm 1$ & $10 \pm 2$ & $4 \pm 3$ \\
\hline & 44 & ALL & $23.52 \pm 2.55$ & $17.36 \pm 6.62$ & $3 \pm 2$ & $3 \pm 1$ & $8 \pm 3$ & $4 \pm 1$ \\
\hline & 22 & $4 \mathrm{~L}$ & $23.74 \pm 3.08$ & $12.99 \pm 5.42$ & $3 \pm 1$ & $2 \pm 3$ & $7 \pm 2$ & $3 \pm 2$ \\
\hline & 44 & $4 \mathrm{~L}$ & $22.29 \pm 2.98$ & $22.62 \pm 6.82$ & $4 \pm 2$ & $6 \pm 3$ & $6 \pm 2$ & $7 \pm 4$ \\
\hline & 22 & $4 \mathrm{E}$ & $22.74 \pm 4.79$ & $16.24 \pm 2.77$ & $2 \pm 1$ & $2 \pm 1$ & $7 \pm 2$ & $4 \pm 1$ \\
\hline & 44 & $4 \mathrm{E}$ & $21.26 \pm 5.04$ & $19.26 \pm 6.33$ & $3 \pm 0.5$ & $3 \pm 1$ & $7 \pm 2$ & $5 \pm 1$ \\
\hline & 22 & $3 \mathrm{~L}$ & $23.86 \pm 2.88$ & $11.20 \pm 3.74$ & $3 \pm 2$ & $3 \pm 2$ & $5 \pm 3$ & $5 \pm 2$ \\
\hline & 44 & $3 \mathrm{~L}$ & $28.00 \pm 4.17$ & $17.02 \pm 2.65$ & $4 \pm 1$ & $2 \pm 1$ & $9 \pm 3$ & $3 \pm 2$ \\
\hline & 22 & $3 \mathrm{E}$ & $26.21 \pm 6.16$ & $16.02 \pm 4.78$ & $4 \pm 2$ & $1 \pm 0.5$ & $6 \pm 3$ & $2 \pm 1$ \\
\hline & 44 & $3 \mathrm{E}$ & $24.53 \pm 5.32$ & $22.51 \pm 2.54$ & $3 \pm 1$ & $5 \pm 2$ & $9 \pm 4$ & $6 \pm 3$ \\
\hline & Significance & NS & $* *$ & $* *$ & $* *$ & $*$ & $*$ & \\
\hline
\end{tabular}

${ }^{\mathrm{z}} \mathrm{L}=$ late, $\mathrm{E}=$ early.

Ns,*,**,**** Nonsignificant or significant at $P \leq 0.05,0.01$, or 0.0005 , respectively.

producers, as evidenced by the low yields under all treatments and from previous yield history records (Table 2). These results indicate that, in low yield situations, $\mathrm{N}$ fertilizers are not advantageous for crop production.

At the same locations where yield was highest with $0 \mathrm{~N}$, fruit rot showed slightly different patterns (Table 5). 'Howes' showed no difference in any of the rots by treatment in any year or at either of the two locations. In Washington, 'McFarlin' only showed a difference in rot at $30 \mathrm{~d}$ in 1992. The 22 rate applied in four applications gave the lowest level of rot. However if $44 \mathrm{~kg} \mathrm{~N} / \mathrm{ha}$ was applied over the same timing, the rot level was highest.

'Early Black' in Massachusetts only showed a difference in yield with different rates and timings in 1 year. The data indicate that the 22 rate gave the best yield when applied in four early season applications (Table 5). 'Early Black' in Massachusetts consistently showed the lowest rot with the $22 \mathrm{~kg} \mathrm{~N}$ rate in four early applications at Bog 1 and five applications at Bog 2. The 44 rate in four early applications was one of the treatments with highest rot at both locations.

In New Jersey, the same cultivar showed a different response. At both locations, in high- and low-yielding beds, applying $\mathrm{N}$ fertilizer at $22 \mathrm{~kg} \cdot \mathrm{ha}^{-1}$ in three early or three late applications resulted in the highest yields on plots that received $\mathrm{N}$ in 1991 and 1992. 'Early Black' at both locations in New Jersey showed the lowest field and storage rot levels in plots receiving $\mathrm{N}$ when $22 \mathrm{~kg}$ $\mathrm{N} / \mathrm{ha}$ was applied in three late applications. Applications of $44 \mathrm{~kg}$ $\mathrm{N} /$ ha in three early applications gave the highest rot at both locations. 
'Stevens' in Wisconsin only showed a significant difference in yield by timing in 1992, the third year of the experiment. In this case, applying the fertilizer at $22 \mathrm{~kg} \mathrm{~N} / \mathrm{ha}$ in five applications throughout the growing season was distinctly the best treatment. Applying $22 \mathrm{~kg} \mathrm{~N} / \mathrm{ha}$ in either five or three early applications were the only treatments with consistently low rot plus high yields in 'Stevens'.

Results with 'Searles' also in Wisconsin, were less clear than those with 'Stevens'. At one location, Bog 9, none of the treatments showed a yield increase. Bog 9 was a mineral-based soil. In the final year, a yield increase was found at the second location (Bog 10). The highest average yield was with the $44 \mathrm{~kg} \mathrm{~N} /$ ha rate over three early or four late applications. That crop year, 1992, was a very cold year in this region of Wisconsin (W. Bland, personal communication). The site that showed the highest yield with two timings at the 44 rate was a muck soil. It is likely that the cold weather resulted in very little $\mathrm{N}$ mineralization from the soil, thus, the higher rate of $\mathrm{N}$ gave the two best treatments. For 'Searles' grown on mineral and organic soils, the 22 rate in three late applications had relatively consistent low rot at 0 and $30 \mathrm{~d}$. Several treatments had high rots, but these were not consistent.

\section{Conclusions}

Across all of the cultivars examined in this study, the yield and rot (field and storage) response to $\mathrm{N}$ suggests that low to moderate $\mathrm{N}$ applications are suitable for cranberries over a variety of conditions. The specific timings of these fertilizers varied by cultivar and state.

In Massachusetts, the evidences for rates and timing on 'Howes' was inconclusive. In the final year at one location, the bed was commercially harvested before research sample collection. However, with the data from this study, no specific timings for $\mathrm{N}$ application can be suggested.

'Early Black' in Massachusetts shows a distinctive rate and timing regime. Applying $22 \mathrm{~kg} \mathrm{~N} / \mathrm{ha}$ in four equal applications in the early season (budbreak, peak bloom, fruit set, and bud set) had the highest yield and lowest rot levels, suggesting that this treatment would be optimal for 'Early Black' production in Massachusetts. The same cultivar in New Jersey, on high- and low-yielding beds, showed optimal productivity at the same $\mathrm{N}$ rate, but applied over three late season applications (peak bloom, fruit set, bud set), since plots fertilized with this treatment had high yields and low rot levels compared to all other fertilized plots. Although, the control $(0 \mathrm{~N})$ treatment had the highest yield on the low-producing bog (Bog 5), the response over the period of this experiment suggests that in the long-term production will decrease if $\mathrm{N}$ is withheld.

In Wisconsin, the evidence for 'Searles' was inconclusive. The treatment that showed highest yield did so only in 1 year. Also, this treatment did not have good fruit quality. Thus, no single treatment suggests itself as the optimal from this experiment. For 'Stevens', the optimal was $22 \mathrm{~kg} \mathrm{~N} /$ ha in five equal applications at budbreak, peak bloom, fruit set, bud set, and preharvest. This treatment showed high yield and low rot and thus optimized cranberry yield.

Overall, the results of this study indicate that cranberries produce optimally when fertilized at the experimental middle rate of $22 \mathrm{~kg} \mathrm{~N} / \mathrm{ha}$. This rate holds consistently across years. However, the optimal timing for the $\mathrm{N}$ applications varied to some extent. Thus, optimal $\mathrm{N}$ fertilizer timing in cranberries varied by cultivar and within growing region and may vary by year.

\section{Literature Cited}

Beckwith, C.S. 1919. The effect of certain nitrogenous and phosphatic fertilizers on the yield of cranberries. Soil Sci. 8:483-490.

Davenport, J.R. and C.J. DeMoranville. 1993. A survey of several soil and physical characteristics of cultivated cranberry bogs in North America. Commun. Soil Sci. Plant Anal. 24 (13-14):1769-1773.

Deubert, K.H. and F.L. Caruso. 1989. Bogs and cranberry bogs in southeastern Massachusetts. Univ. of Mass. Res. Bul. 727.

Eaton, G.W. 1971. Effect of N, P, and K fertilizer applications on cranberry leaf nutrient composition, fruit color, and yield in a mature bog. J. Amer. Soc. Hort. Sci. 96:430-433.

Eck, P. 1976. Relationship of nitrogen nutrition of 'Early Black' cranberry to vegetative growth, fruit yield, and quality. J. Amer. Soc. Hort Sci. 101:375-377.

Fuleki, T. and F.J. Francis. 1968. Quantitative methods for anthocyanins: I. Extraction and determination of total anthocyanin in cranberries. J. Food Sci. 33:72-77.

Hart, J.M., A. Poole, K.L. Wilder, and B.C. Strik. 1990. Nitrogen rate and timing affect on cranberry yield and yield components. HortScience 25:1148.

Peterson, B.S., C.E. Cross, and N. Tilden. 1968. The cranberry industry in Massachusetts. Mass. Dept. Agr. Bul. 201.

U.S. Department of Agriculture. 1990. New England agricultural statistics: Cranberries. USDA Natl. Agr. Stat. Serv., Concord, N.H. 